\title{
Effects of Dietary Cholesterol on Cholesterol and Bile Acid Homeostasis in Patients with Cholesterol Gallstones
}

Fred Kern, Jr.

Division of Gastroenterology and Hepatobiliary Research Center, University of Colorado School of Medicine, Denver, Colorado 80262

\begin{abstract}
We examined changes in cholesterol and bile acid metabolism produced by dietary cholesterol in gallstone subjects and matched controls. Healthy women were recruited and, after confirming the presence or absence of radiolucent gallstones, they were studied on regular diets and again on the same diet supplemented with five eggs daily for 15-18 d. Studies included plasma lipids, lipoproteins and apolipoproteins, dietary records, cholesterol absorption, cholesterol synthesis, plasma clearance of chylomicron remnants, biliary lipid composition, and secretion and bile acid kinetics. On low cholesterol, gallstone subjects absorbed a slightly lower fraction of dietary cholesterol, synthesized more cholesterol, and had smaller bile acid pools and faster fractional turnover rate (FTR) of bile acids. On high cholesterol, the fraction of cholesterol absorbed decreased in both groups and cholesterol synthesis decreased, especially in the gallstone group. Biliary cholesterol secretion increased in the gallstone group only. FTR of bile acids did not change in either group. Bile acid synthesis and pool tended to increase ( $P=N S$ ) in the controls, but in gallstone subjects, synthesis and pool size decreased. We concluded that in gallstone subjects cholesterol and bile acid homeostasis is significantly altered, and that increasing dietary cholesterol increases biliary cholesterol secretion and decreases bile acid synthesis and pool, changes associated with cholesterol gallstone formation. (J. Clin. Invest. 1994. 93:1186-1194.) Key words: cholesterol metabolism - cholesterol, dietary • cholelithiasis • bile acids and salts $\bullet$ lipids, biliary secretion
\end{abstract}

\section{Introduction}

Cholesterol gallstones occur predominantly in Western cultures and are generally believed to be related to diet, especially excess cholesterol, but the effect of dietary cholesterol on cholesterol in the bile has not been clearly established. Published studies, each involving only a small number of subjects, are contradictory (1-5), showing that dietary cholesterol either increases or does not affect cholesterol saturation of gallbladder bile. The rate of biliary secretion of cholesterol has not been described in most of these reports. In a recent study we found

Address correspondence to Dr. F. Kern, Jr., Division of Gastroenterology and Hepatobiliary Research, University of Colorado School of Medicine, Denver, CO 80262.

Received for publication 4 May 1993 and in revised form 27 September 1993.

J. Clin. Invest.

(c) The American Society for Clinical Investigation, Inc. 0021-9738/94/03/1186/09 \$2.00

Volume 93, March 1994, 1186-1194 that increasing dietary intake of cholesterol $\sim 1,600 \mu \mathrm{mol}$ $(610 \mathrm{mg})$ per day increased the secretion of all biliary lipidsbile acid, phospholipid, and cholesterol-but did not alter the cholesterol saturation index (6). The homeostatic and regulatory responses to dietary cholesterol, including secretion of cholesterol into bile, have been extensively studied in recent years, but such studies have not been published in patients with gallstones. The homeostatic and regulatory responses, including cholesterol absorption, cholesterol synthesis, cholesterol esterification, biliary excretion of cholesterol, LDL receptor activity, and biosynthesis of bile acids, are complex and are known to vary considerably among individuals (7-15). In recent years noninvasive techniques, not requiring lengthy hospitalization for the accurate measurement of these processes, have been developed.

The purpose of this study was to test the hypothesis that the metabolic responses to dietary cholesterol are different in subjects with and without gallstones, and that, when dietary cholesterol is increased, biliary secretion of cholesterol is greater in those with gallstones. The hypothesis was confirmed, and other important metabolic differences between gallstone and control subjects were identified. These include a $44 \%$ greater rate of sterol synthesis by freshly isolated peripheral mononuclear cells during intake of the regular diet and a decrease in bile acid synthesis and pool in response to enhanced dietary cholesterol intake in gallstone subjects. Bile acid kinetics did not change significantly in control subjects fed the same diet.

\section{Methods}

Subjects. This study was approved by the Human Subject Committee of the University of Colorado School of Medicine. All subjects were paid volunteers and gave written informed consent. Eight women with asymptomatic radiolucent gallstones and eight women control subjects without gallstones were studied. In so far as possible, control subjects were matched to gallstone subjects for age, weight, and body mass index (BMI) 1 -weight/height squared (Table I). The mean $( \pm$ SD) age of the control and gallstone subjects was $34.8 \pm 3.1$ and $46.9 \pm 3.4 \mathrm{yr}$, respectively $(P<0.05)$ (Table I). The mean weight of the controls was $72.7 \pm 4.1 \mathrm{~kg}$ and gallstone subjects, $71.0 \pm 4$.5. Four subjects in each group were obese; i.e., their weight was $>120 \%$ of ideal body weight (16). The mean BMI was $28.0 \pm 7.1 \mathrm{~kg} / \mathrm{m}^{2}$ in controls and $27.7 \pm 1.6$ in gallstone subjects.

The subjects were healthy and were not taking medications. None had had symptoms of biliary tract disease for at least 3 mo. Appropriate laboratory studies were performed to exclude hematologic, liver, biliary tract, and metabolic disease. Most had normal plasma lipids and lipoproteins, including plasma total, LDL and HDL cholesterol, and triglyceride. Two women in each group had total plasma cholesterol levels above $5.18 \mu \mathrm{M}(200 \mathrm{mg} / \mathrm{dl})$. Plasma apolipoproteins A-I and B

1. Abbreviations used in this paper: BMI, body mass index; CSI, cholesterol saturation index; FTR, fractional turnover rate. 
Table I. Characteristics of Study Subjects

\begin{tabular}{lcccccc}
\hline & \multicolumn{2}{c}{$\operatorname{CONTROL}(n=8)$} & & \multicolumn{2}{c}{$\operatorname{GALLSTONE}(n=8)$} & \\
\cline { 2 - 3 } \multicolumn{1}{c}{ Characteristic } & Mean \pm SEM & Range & & Mean \pm SEM & Range & $P$ \\
\hline Age & $34.8 \pm 3.1$ & $27-48$ & & $46.9 \pm 3.4$ & $32-60$ & $<0.05$ \\
Weight $(\mathrm{kg})$ & $72.7 \pm 4.1$ & $59-93$ & $71.0 \pm 4.5$ & $49-95$ & NS \\
Ideal weight $(\%)$ & $123.7 \pm 7.6$ & $94-157$ & & $121.5 \pm 6.4$ & $95-156$ & NS \\
BMI $\left(\mathrm{kg} / \mathrm{ht}^{2}\right)$ & $28.0 \pm 2.1$ & $20.7-36.5$ & $27.7 \pm 1.6$ & $22.8-34.9$ & NS
\end{tabular}

were also normal. Experimental subjects had gallstones identified on ultrasound examination and shown by oral cholecystography to be radiolucent and noncalcified. Oral cholecystograms and/or ultrasound failed to show stones in the control subjects.

Study protocol. Each subject was studied on her regular diet and again after 15-18 d on the same diet supplemented with five eggs a day. Plasma lipids and apolipoproteins A-I and B, measured by standard clinical laboratory techniques, were determined three times before beginning the control period studies and again at the completion of each study period. The following were measured at the end of each dietary period: cholesterol absorption, cholesterol synthesis and esterification, plasma clearance of retinyl palmitate, bile acid kinetics, composition of duodenal bile after gallbladder stimulation, and biliary lipid secretion.

Diet. The Clinical Research Center dietician took a careful dietary history and instructed the subjects in the maintenance of a diary of daily food intake (17). The diet and diary were reviewed periodically by the dietician, who calculated with the aid of a computer program (Food Processor II, ESHA Research, Salem, OR) the mean daily intake of calories, cholesterol, saturated, mono- and polyunsaturated fat, and other nutrients. The additional five eggs were prepared and eaten in any manner preferred by the subjects. Since the reported cholesterol content of eggs varies, we measured cholesterol in locally purchased large eggs. The cholesterol was extracted from the yolks, hydrolyzed, and derivatized, and the concentration was measured by gel liquid chromatography. The mean was $604.9 \pm 34.2 \mu \mathrm{mol}(233.5 \pm 13.2 \mathrm{mg})$ per egg.

Procedures. All procedures have been in use in our laboratory for a number of years and have been described $(6,18)$. Cholesterol absorption was measured using the isotope ratio method (19) which required the simultaneous oral administration of $2 \mu \mathrm{Ci}$ of $\left[4-{ }^{14} \mathrm{C}\right]$ cholesterol and the intravenous administration of $2 \mu \mathrm{Ci}\left[1,2-{ }^{3} \mathrm{H}\right]$ cholesterol. After 48 and $72 \mathrm{~h}$, samples of plasma were drawn, the isotope ratio was determined, and the percent cholesterol absorbed was calculated. Cholesterol synthesis was estimated from the incorporation of $\left[2-{ }^{14} \mathrm{C}\right]-$ acetate (New England Nuclear, Boston, MA) into sterols by freshly isolated peripheral mononuclear cells (20). Plasma clearance of retinyl palmitate was measured as previously described $(6,18)$. Retinyl palmitate was given by mouth and plasma concentration of retinyl palmitate was measured at intervals for $24 \mathrm{~h}$. The concentration was plotted against time, and the area under the curve was taken as the hepatic clearance of chylomicron remnants $(6,18,21,22)$.

Biliary lipid composition and secretion rates were measured by established procedures and methods, described in detail by us and others $(23,24)$. After insertion of a nasoduodenal tube, a sample of duodenal content, "hepatic bile," was obtained and gallbladder contraction was stimulated by an intravenous infusion of the octapeptide of cholecystokinin (CCK; Squibb CCK-8, $0.02 \mu \mathrm{g} / \mathrm{kg}$ per h) and a sample of concentrated bile "gallbladder bile" was collected. Bile acid distribution was measured by gel liquid chromatography and the concentration of bile acid, phospholipids, and cholesterol was determined by previously described methods. The cholesterol saturation of each sample was calculated. A liquid formula test meal, containing [14C]PEG 4000 as nonabsorbable marker, was then infused intraduodenally for $8 \mathrm{~h}$. Samples were collected from the duodenum continually and pooled at 30 min intervals. The concentration of bile acid, phospholipid, choles- terol, and ${ }^{14} \mathrm{C}$ radioactivity was determined in all samples by described methods $(18,21)$ and the secretion rates of each lipid was calculated.

Bile acid kinetics were measured using stable isotopes and gas chromatography/mass spectrometry analysis of serum bile acids (25). 13cholic and 13-chenodeoxycholic acids (Merck, Sharp \& Dohme, Montreal) were given orally, and blood samples were obtained daily at the same time for 4-5 d. Bile acids were extracted and derivatized, and molar ratios of labeled to unlabeled bile acids in each sample were determined by selected ion monitoring. The fractional turnover rate (FTR), pool sizes, and synthesis rates of the primary bile acid were calculated (25). The deoxycholate pool size was determined by comparing the intensity of its ion to the intensity of the chenodeoxycholate ion (6).

Statistical methods. Results are reported as the means \pm SEM. Differences in means between control and gallstone subjects and in the percent change from low to high cholesterol diet were analyzed by Student's $t$ test. Differences within groups on and off the high-cholesterol diet were analyzed by paired $t$ tests. The relationships between variables were analyzed by linear regression.

\section{Results}

\section{Differences between the controls and gallstone subjects}

Dietary intake. The dietary intake of cholesterol, calories, and fat, and the ratio of polyunsaturated to saturated fatty acids and other nutrients (not shown) were similar in the two groups in both study periods ( Table II). Cholesterol intake in the control subjects was $585 \pm 85 \mu \mathrm{mol}(226 \pm 33 \mathrm{mg})$ on the regular diet, compared with $548 \pm 91 \mu \mathrm{mol}(211 \pm 35 \mathrm{mg})$ in the gallstone group $(P=\mathrm{NS})$. On the high-cholesterol diet, it was $2,978 \pm 40 \mu \mathrm{mol}(1,150 \pm 16 \mathrm{mg})$ in the controls and $3,010 \pm 50$ $\mu \mathrm{mol}(1,162 \pm 19 \mathrm{mg})$ in the gallstone group $(P=\mathrm{NS})$.

Plasma lipids. Lipids and apolipoproteins were similar in the two groups of subjects (Table III).

Cholesterol absorption. On the low-cholesterol diet the percent cholesterol absorption tended to be less in the gallstone subjects $(52.7 \pm 1.8 \%$ vs. $60.4 \pm 3.9 \%$ in the controls; $P=0.08$ ); on the high-cholesterol intake, mean absorption was similar in the two groups of subjects $(43.1 \pm 1.4$ vs. $49.0 \pm 4.3)$. The absolute amount of cholesterol absorbed (dietary cholesterol multiplied by percent absorbed) was $20 \%$ less in the gallstone subjects on the control diet $(282.5 \pm 44$ vs. $353.5 \pm 55, P<0.05)$ and $11 \%$ less $(P=\mathrm{NS})$ on the high-cholesterol diet. Fig. 1 shows the changes in fraction absorbed for individual subjects produced by the high-cholesterol diet.

Table II. Mean Daily Dietary Intake

\begin{tabular}{lcccc}
\hline & Calories & Cholesterol & Fat & P/S* \\
\hline & & $\mu m o l$ & $g$ & \\
Control subjects & & & & \\
Low-CH diet & $1716 \pm 145$ & $585 \pm 85$ & $68 \pm 7$ & $0.61 \pm 0.05$ \\
High-CH diet & $1568 \pm 96$ & $2978 \pm 41$ & $76 \pm 5$ & $0.59 \pm 0.04$ \\
$P$ & NS & $<0.0001$ & NS & NS \\
Gallstone subjects & & & & \\
Low-CH diet & $1425 \pm 168$ & $548 \pm 92$ & $57 \pm 11$ & $0.68 \pm 0.09$ \\
High-CH diet & $1343 \pm 71$ & $3010 \pm 50$ & $66 \pm 5$ & $0.65 \pm 0.08$ \\
$P$ & NS & $<0.0001$ & NS & NS \\
& & & & \\
\hline
\end{tabular}

Values are given as mean \pm SEM. None of the differences between control and gallstone subjects is statistically significant. Abbreviation: $\mathrm{CH}$, cholesterol. * Polyunsaturated/saturated ratio. 


\begin{tabular}{|c|c|c|c|c|c|c|}
\hline & \multicolumn{3}{|c|}{ Cholesterol } & \multirow[b]{2}{*}{ TGL } & \multicolumn{2}{|c|}{ Apolipoproteins } \\
\hline & Total & LDL & HDL & & A-1 & B \\
\hline & & $m M$ & & $m g / d l$ & & \\
\hline \multicolumn{7}{|l|}{ Control subjects } \\
\hline Low-CH diet & $4.38 \pm 0.24$ & $2.78 \pm 0.21$ & $1.08 \pm 0.09$ & $103.8 \pm 15.3$ & $133.7 \pm 9.0$ & $83.1 \pm 8.5$ \\
\hline High-CH diet & $4.52 \pm 0.25$ & $2.89 \pm 0.20$ & $1.13 \pm 0.08$ & $96.1 \pm 13.7$ & $140.0 \pm 11.4$ & $80.0 \pm 7.5$ \\
\hline$P$ & NS & NS & NS & 0.07 & NS & NS \\
\hline \multicolumn{7}{|l|}{ Gallstone subjects } \\
\hline Low-CH diet & $5.03 \pm 0.30$ & $3.22 \pm 0.22$ & $1.33 \pm 0.16$ & $97.0 \pm 13.7$ & $155.4 \pm 12.8$ & $80.5 \pm 4.9$ \\
\hline High-CH diet & $4.81 \pm 0.37$ & $3.05 \pm 0.22$ & $1.28 \pm 0.11$ & $93.6 \pm 13.0$ & $154.7 \pm 14.4$ & $80.1 \pm 5.5$ \\
\hline$P$ & NS & NS & NS & 0.05 & NS & NS \\
\hline
\end{tabular}

Values are given as mean \pm SEM. Neither the differences between mean values for control and gallstone subjects nor the differences between percent change is statistically significant. Abbreviations: $\mathrm{CH}$, cholesterol; TGL, triglycerides.

Cholesterol synthesis. The rate of cholesterol synthesis was consistently higher in the gallstone subjects on both diets (Fig. $2)$. On the low-cholesterol diet it was $73.4 \pm 2.7 \mathrm{pmol} / 10^{7} \mathrm{cells}$ per $\mathrm{h}$ in the gallstone group and $47.6 \pm 2.6$ in the controls $(P$ $<0.001)$, and on high cholesterol it was respectively $58.3 \pm 3.5$ vs. $44.3 \pm 2.4(P<0.03)$. Chylomicron remnant clearance was the same in both groups on both diets.

Biliary lipids. On the low-cholesterol diet, the cholesterol saturation index (CSI) and the secretion rate of biliary lipids were the same in the two groups (Table IV). The molar percent cholesterol in gallbladder bile was slightly higher in the gallstone subjects and the rate of biliary secretion of cholesterol was slightly higher in the control group, but neither difference was significant. On the high cholesterol intake, the molar percent cholesterol was $42 \%$ higher in the gallstone group ( $7.92 \pm 1.38$ vs. $5.58 \pm 0.41 ; P=0.08)$, but the secretion rate of cholesterol did not differ significantly between the groups. Bile acid and phospholipid secretion rates as well as the CSI of hepatic and gallbladder bile were similar. The mean distribution of bile acids in gallbladder bile did not differ between groups.

Bile acid kinetics. Bile acid kinetics differed in the two groups (Table V). FTR of both bile acids were greater in the
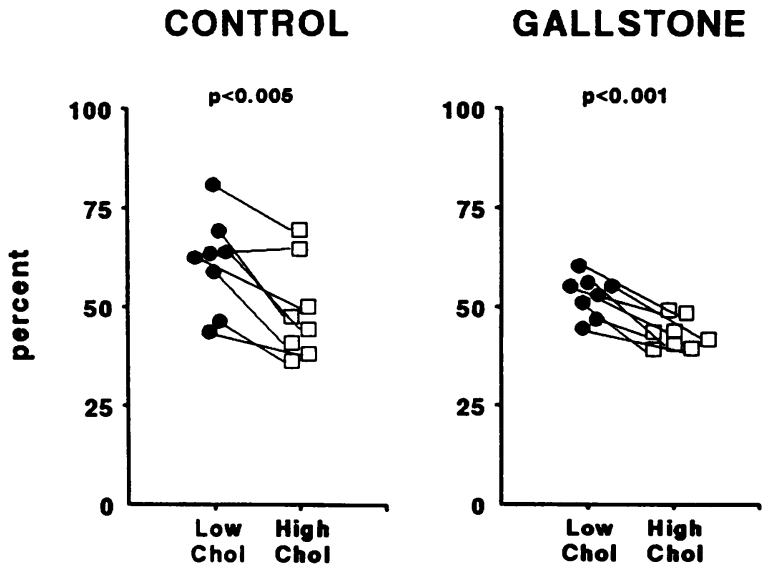

Figure 1. Fraction of cholesterol absorbed in control and gallstone subjects on both diets. The high-cholesterol diet caused a highly significant decrease in both groups. Each point represents a single subject. gallstone subjects on both diets. The pools of both bile acids were smaller in those with gallstones on both diets, especially the high cholesterol diet; on the high cholesterol diet $(P=0.08$ for chenodeoxycholic acid, $<0.025$ for cholic acid, $<0.05$ for deoxycholic acid, and $<0.02$ for total bile acid). The synthesis rates of each primary bile acid was slightly, but not signifcantly, smaller in the gallstone group on both diets. The total bile acid synthesis was $42 \%$ smaller in the gallstone subjects on the high cholesterol diet $(P<0.07)$.

\section{Responses to the high-cholesterol diet}

Plasma lipids. No plasma lipid or apolipoprotein was altered by $15-18 \mathrm{~d}$ of the high-cholesterol diet except the plasma triglyceride, which decreased slightly on the high-cholesterol diet in both groups (Table II). Neither total nor LDL cholesterol increased.

Cholesterol absorption and synthesis. The percent cholesterol absorption decreased in almost every subject when the cholesterol intake was increased (Fig. 1). The mean decreased from $60.1 \pm 3.9 \%$ to $49.0 \pm 4.3 \%(P<0.01)$ in the controls and
CONTROL

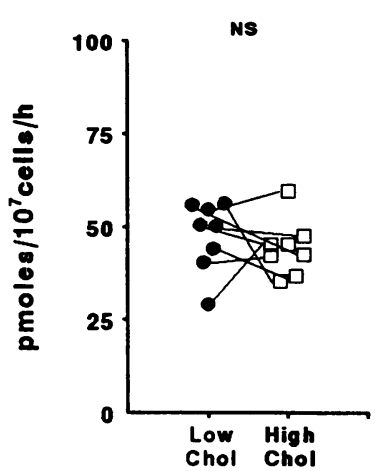

GALLSTONE

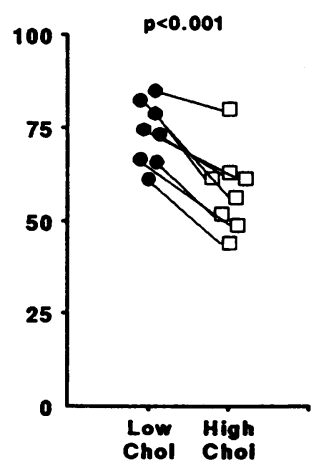

Figure 2. Cholesterol synthesis measured by incorporation of labeled acetate into sterols in freshly isolated peripheral mononuclear cells. Each point is a single subject. In the controls, there was no consistent change on the high-cholesterol diet. Synthesis was clearly greater in the gallstone subjects on the low-cholesterol diet and it decreased in each one on the high cholesterol intake. 


\begin{tabular}{|c|c|c|c|c|c|c|c|c|}
\hline & \multicolumn{2}{|c|}{ CSI } & \multirow[b]{2}{*}{ BA } & \multirow[b]{2}{*}{ PL } & \multirow[b]{2}{*}{$\mathrm{CH}$} & \multicolumn{3}{|c|}{ Secretion } \\
\hline & Hep & GB & & & & BA & PL & $\mathrm{CH}$ \\
\hline & & & & $M \%$ & & & $\mu \mathrm{mol} / \mathrm{kg} \mathrm{per}$ & \\
\hline \multicolumn{9}{|l|}{ Control subjects } \\
\hline Low-CH diet & $\begin{array}{c}1.563 \\
(0.248)\end{array}$ & $\begin{array}{c}1.158 \\
(0.060)\end{array}$ & $\begin{array}{l}77.60 \\
(1.08)\end{array}$ & $\begin{array}{l}16.67 \\
(0.82)\end{array}$ & $\begin{array}{c}5.54 \\
(0.35)\end{array}$ & $\begin{array}{l}26.86 \\
(5.64)\end{array}$ & $\begin{array}{c}5.60 \\
(0.90)\end{array}$ & $\begin{array}{c}1.88 \\
(0.26)\end{array}$ \\
\hline High-CH diet & $\begin{array}{c}1.601 \\
(0.175)\end{array}$ & $\begin{array}{c}1.178 \\
(0.067)\end{array}$ & $\begin{array}{l}75.08 \\
(1.78)\end{array}$ & $\begin{array}{l}19.09 \\
(1.16)\end{array}$ & $\begin{array}{c}5.58 \\
(0.41)\end{array}$ & $\begin{array}{l}25.70 \\
(5.28)\end{array}$ & $\begin{array}{c}5.67 \\
(0.66)\end{array}$ & $\begin{array}{c}1.85 \\
(0.22)\end{array}$ \\
\hline$P$ & NS & NS & NS & NS & NS & NS & NS & NS \\
\hline \multicolumn{9}{|l|}{ Gallstone subjects } \\
\hline Low-CH diet & $\begin{array}{c}2.000 \\
(0.445)\end{array}$ & $\begin{array}{c}1.180 \\
(0.093)\end{array}$ & $\begin{array}{l}75.47 \\
(1.71)\end{array}$ & $\begin{array}{l}17.61 \\
(1.07)\end{array}$ & $\begin{array}{c}6.94 \\
(0.82)\end{array}$ & $\begin{array}{l}19.57 \\
(3.24)\end{array}$ & $\begin{array}{c}4.24 \\
(0.42)\end{array}$ & $\begin{array}{l}1.60 \\
(.18)\end{array}$ \\
\hline High-CH diet & $\begin{array}{c}1.427 \\
(0.335)\end{array}$ & $\begin{array}{c}1.237 \\
(0.478)\end{array}$ & $\begin{array}{l}73.46 \\
(0.55)\end{array}$ & $\begin{array}{l}18.60 \\
(0.68)\end{array}$ & $\begin{array}{c}8.12^{*} \\
(1.25)\end{array}$ & $\begin{array}{l}20.73 \\
(2.08)\end{array}$ & $\begin{array}{c}5.27 \\
(0.95)\end{array}$ & $\begin{array}{c}2.01 \\
(0.39)\end{array}$ \\
\hline$P$ & NS & NS & NS & NS & 0.08 & NS & NS & $<0.05$ \\
\hline
\end{tabular}

Values are given as mean \pm SEM. Abbreviations: BA, bile acid; $\mathrm{CH}$, cholesterol; GB, gallbladder; Hep, hepatic; PL, phospholipid. ${ }^{*}$ Differs from molar percent cholesterol in control group on high-cholesterol diet $0.05<P<0.08$. The difference in percent change in molar percent cholesterol between the controls $(<1.0 \%)$ and the gallstones subjects $(25 \%)$ was not quite significant. There are no other statistically significant differences between control and gallstone subjects.

from $52.7 \pm 1.8 \%$ to $43.1 \pm 1.3 \%(P<0.001)$ in the gallstone patients. The rate of sterol synthesis (Fig. 2) did not change significantly in the controls $\left(47.3 \mathrm{pmol} / 10^{7}\right.$ cells per $\mathrm{h}$ on the low diet, 43.2 on the high diet), but in the gallstone group synthesis decreased from $73.4 \pm 3.0$ to $58.3 \pm 3.9(P<0.001)$. The difference between the groups in percent change in absorption and synthesis was not significant. The rate of synthesis decreased as the amount of cholesterol absorbed increased in the gallstone group only ( $r=0.72, P<0.05)$ (data not shown). Retinyl palmitate clearance rate was unaffected by diet in either group.

Biliary lipids. The high cholesterol diet did not significantly influence biliary lipid composition or secretion in control subjects (Fig. 3 and Table IV). In the gallstone subjects, however, the diet caused an increase in the rate of cholesterol secretion in most patients $(1.60 \pm 0.18 \mu \mathrm{mol} / \mathrm{h}$ on the low diet, $2.01 \pm 0.39$ on the high, $P<0.05)$. There was also an increase in mean molar percent cholesterol (from $6.84 \pm 0.82$ to $7.92 \pm 1.33, P$ $<0.08$ ), in the same group, but the CSI of gallbladder bile did not change significantly ( 1.1 vs. 1.27$)$. There were no significant differences in rate of secretion of bile acid or phospholipid in either group (Fig. 4).

\section{Bile acid kinetics}

Dietary cholesterol affected bile acid kinetics quite differently in the two groups (Fig. 4 and Table V). In the controls, there was a trend toward an increase in bile acid synthesis and pool size. Cholic acid and total bile acid synthesis increased in six of

Table V. Bile Acid Kinetics

\begin{tabular}{|c|c|c|c|c|c|c|c|c|c|}
\hline & \multicolumn{3}{|c|}{ Chenodeoxycholic acid } & \multicolumn{3}{|c|}{ Cholic acid } & \multirow{2}{*}{$\begin{array}{l}\text { Deoxycholic } \\
\text { acid pool }\end{array}$} & \multicolumn{2}{|c|}{ Total bile acid } \\
\hline & FTR & Pool & Synthesis & FTR & Pool & Synthesis & & Pool & Synthesis \\
\hline & days $^{-1}$ & $\mu m / k g$ & $\mu \mathrm{m} / \mathrm{kg}$ per $d$ & days $^{-1}$ & $\mu m / k g$ & $\mu m / k g$ per $d$ & $\mu m / k g$ & $\mu m / k g$ & $\mu \mathrm{m} / \mathrm{kg}$ per $d$ \\
\hline \multicolumn{10}{|l|}{ Control subjects } \\
\hline Low-CH diet & $0.157 \pm 0.014$ & $20.58 \pm 2.36$ & $3.24 \pm 0.23$ & $0.165 \pm 0.028$ & $33.22 \pm 8.76$ & $5.71 \pm 0.90$ & $13.50 \pm 2.21$ & $72.38 \pm 6.44$ & $8.95 \pm 0.84$ \\
\hline High-CH diet & $0.168 \pm 0.013$ & $24.52 \pm 3.57$ & $3.58 \pm 0.30$ & $0.189 \pm 0.022$ & $35.16 \pm 5.85$ & $6.50 \pm 0.52$ & $18.03 \pm 1.86$ & $74.25 \pm 9.78$ & $10.07 \pm 0.50$ \\
\hline Percent change & $11.6 \pm 9.3$ & $8.1 \pm 10.3$ & $20.5 \pm 10.3$ & $30.8 \pm 20.7$ & $8.1 \pm 6.5$ & $15.8 \pm 11.5$ & $52.5 \pm 36.1$ & $7.9 \pm 8.9$ & $16.7 \pm 10.7$ \\
\hline$P$ & NS & NS & NS & NS & NS & NS & NS & NS & NS \\
\hline \multicolumn{10}{|l|}{ Gallstone } \\
\hline Low-CH diet & $0.275 \pm 0.050$ & $14.33 \pm 1.94$ & $3.48 \pm 0.31$ & $0.309 \pm 0.063$ & $21.50 \pm 2.88$ & $5.91 \pm 0.61$ & $9.37 \pm 1.49$ & $44.87 \pm 5.06$ & $9.25 \pm 0.82$ \\
\hline High-CH diet & $0.284 \pm 0.072$ & $13.23 \pm 2.44$ & $2.91 \pm 0.33$ & $0.321 \pm 0.068$ & $18.21 \pm 3.53$ & $4.71 \pm 0.85$ & $8.11 \pm 1.36$ & $39.55 \pm 6.16$ & $7.62 \pm 0.94$ \\
\hline Percent change & $0.2 \pm 5.0$ & $-9.3 \pm 8.9$ & $-11.9 \pm 6.8$ & $2.6 \pm 6.5$ & $-20.3 \pm 9.0$ & $-21.0 \pm 7.5$ & $-10.8 \pm 13.1$ & $-14.7 \pm 8.1$ & $-17.3 \pm 6.9$ \\
\hline$P$ & NS & NS & NS & NS & 0.05 & $<0.05$ & NS & 0.05 & $<0.05$ \\
\hline \multicolumn{10}{|l|}{$\begin{array}{l}\text { Control vs. gallstone } \\
\text { ( } P \text { value })\end{array}$} \\
\hline Low-CH diet & $<.05$ & 0.08 & NS & $<.05$ & NS & NS & NS & NS & NS \\
\hline High-CH diet & 0.08 & 0.08 & NS & 0.08 & $<0.025$ & NS & $<0.05$ & $<0.02$ & 0.07 \\
\hline Percent change* & NS & NS & $<0.05$ & NS & $<0.05$ & $<0.05$ & NS & NS & $<0.05$ \\
\hline
\end{tabular}

Values are given as mean \pm SEM. Abbreviation: $\mathrm{CH}$, cholesterol. * Low- to high-cholesterol diet. 
CONTROL
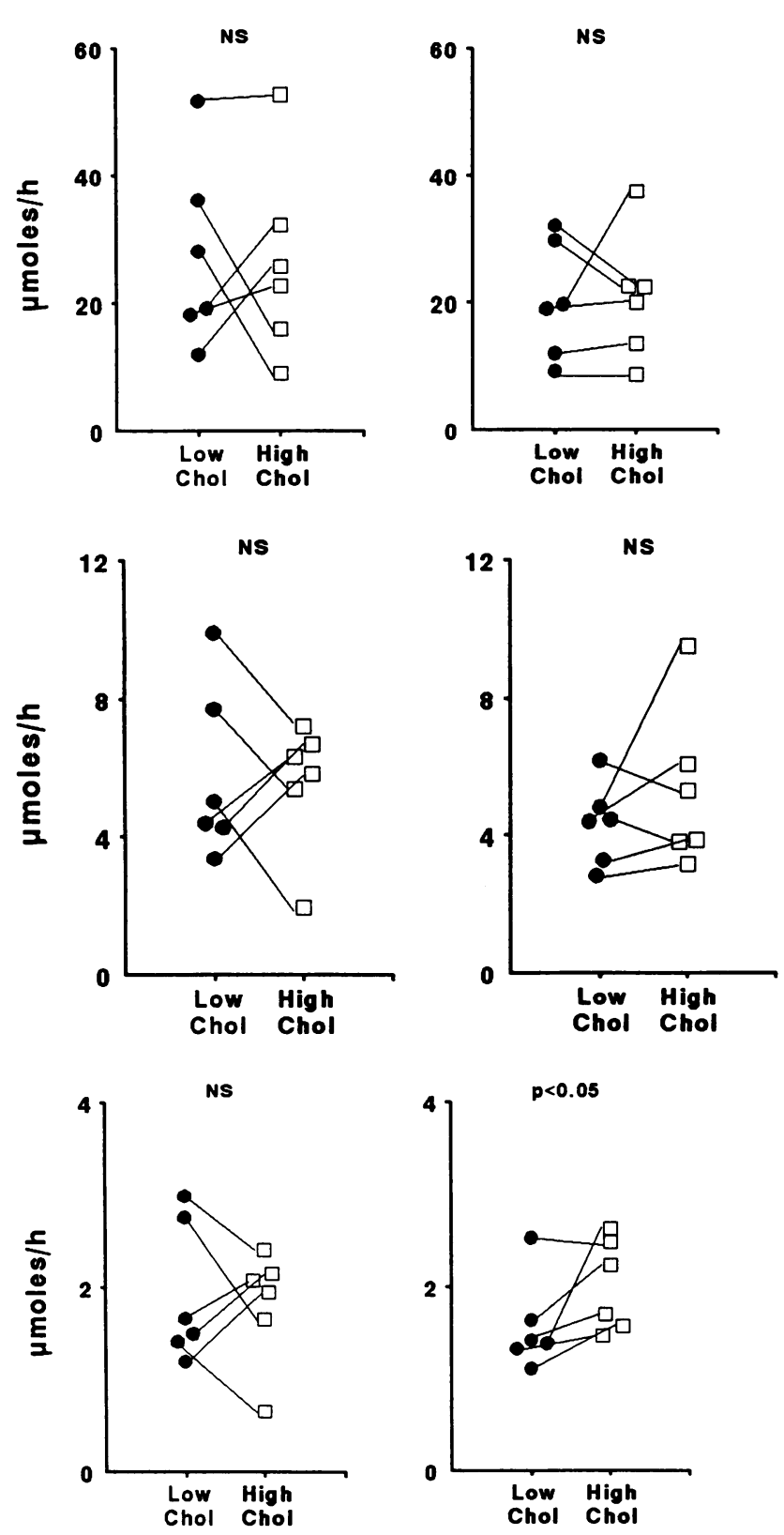

Figure 3. Biliary secretion rates of bile acid (top), phospholipid (middle), and cholesterol (bottom) in both groups on both diets. There was a slight, but significant, increase in the rate of cholesterol secretion in the gallstone subjects on the high-cholesterol diet. No other significant changes were found. There is a wide spread of all data points.

these eight subjects, but the changes were not statistically significant. In the gallstone subjects (Fig. 4), however, the cholic acid pool decreased from $21.5 \pm 2.88 \mu \mathrm{mol} / \mathrm{kg}$ to $18.21 \pm 3.53(P$ $<0.05)$, and its synthesis rate decreased from $5.91 \pm 0.61$ $\mu \mathrm{mol} / \mathrm{kg}$ per d to $4.71 \pm 0.85, P<0.05)$. There were similâsignificant decreases in total bile acid synthesis and pool. There were no consistent or significant changes in chenodeoxycholic acid pool or synthesis, in deoxycholic acid pool or in the FTR of either bile acid. The slight increase in bile acid synthesis in

\section{CA SYN}

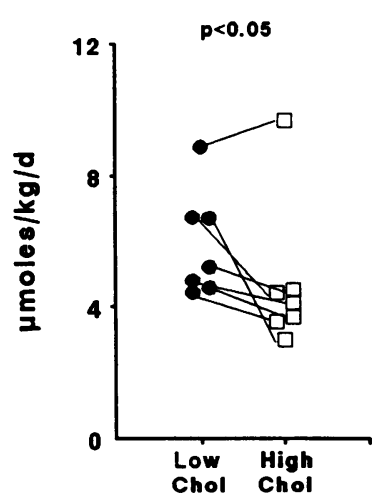

CA POOL
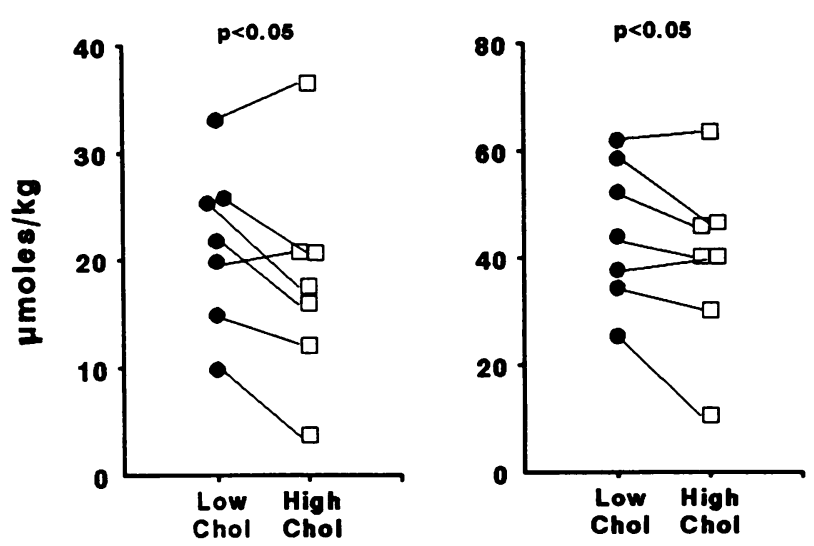

Figure 4. Top: Cholic acid (CA) and total bile acid (BA) synthesis in the gallstone subjects on the low- and high-cholesterol diets. Synthesis decreased in all subjects but one. Each point is an individual subject. Bottom: Cholic and total bile acid pool also decreased on the high-cholesterol diet.

the controls differed significantly from the slight decrease in bile acid synthesis in the gallstone group $(P<0.05)$.

The major differences in cholesterol and bile acid metabolism caused by the added dietary cholesterol in the two groups are summarized in Fig. 5. Cholesterol absorption efficiency decreased $18 \%$ in each group. Cholesterol synthesis decreased $21 \%$ in gallstone subjects and only $7 \%$ in controls. Molar percent cholesterol in bile and biliary cholesterol secretion increased $17 \%$ and $26 \%$, respectively, in the gallstone group and decreased slightly in controls. Total bile acid synthesis and pool decreased $17 \%$ and $15 \%$, respectively, in gallstone subjects and increased $17 \%$ and $8 \%$, respectively, in controls.

\section{Discussion}

Although the pathogenesis of cholesterol gallstones is complex and involves a number of recognized steps, the first, the hepatic secretion of bile that contains excess cholesterol, is a necessary condition for gallstone formation. Known risk factors for the secretion of excess cholesterol into bile include age, obesity, female gender, and female sex hormone administration (26). In addition, factors that include total calories and cholesterol 


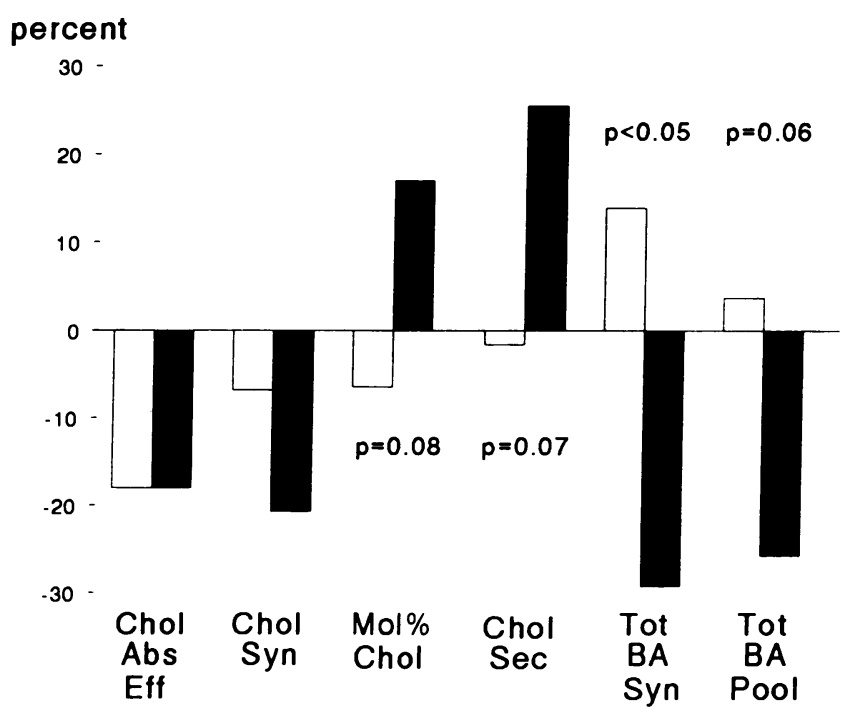

Figure 5. This bar graph summarizes the major changes induced by the high-cholesterol diet in both groups of subjects. Controls are shown in open bars and gallstone subjects in black bars. In the gallstone subjects, dietary cholesterol produced a greater decrease in cholesterol synthesis, an increase in molar percent cholesterol in bile, an increase in cholesterol secreted in bile, and decreases in cholic acid and total bile acid synthesis.

may be risk factors contributing to the high prevalence of cholesterol gallstones in the Western world (27). Epidemiologic studies support this belief, but direct studies of the effect of dietary cholesterol on biliary lipid composition and secretion and on bile acid metabolism are limited. Table VI, which summarizes the reported studies of dietary cholesterol, shows that biliary cholesterol saturation increased in three and did not change in two of five studies in which subjects were fed highcholesterol diets.

The goal of this study was to gain insight into the pathogenesis of cholesterol gallstones by studying the homeostatic and regulatory responses triggered by dietary cholesterol in groups of subjects with and without radiolucent gallstones. Using methods developed in the past $15 \mathrm{yr}$, we studied most of these responses, except LDL receptor activity, in nonhospitalized, healthy individuals. The homeostatic responses to increased dietary cholesterol include decrease in intestinal absorption of cholesterol, inhibition of cholesterol synthesis, down-regulation of LDL receptor uptake, increase in cholesterol esterifica- tion, increase in biliary secretion of cholesterol, and an increase in hepatic conversion of cholesterol to bile acids $(8,12,27-$ 29 ). These responses vary greatly among animal species and in man there is marked individual heterogeneity in each response. It seems reasonable to hypothesize that individuals with cholesterol gallstones who eat a high cholesterol diet might secrete into the bile more cholesterol than those without stones.

All of the subjects were women simply because we were unable to attract male volunteers. None was taking female sex hormones or other medications known to affect hepatobiliary function or lipid metabolism. They were well matched for weight and body mass index, variables thought to influence cholesterol and bile acid metabolism. The controls were younger than the gallstone group (Table I). There were, however, no significant correlations in either group of subjects (or the groups combined) between age and any measured variable including cholesterol absorption and synthesis, biliary lipid composition and secretion, CSI, bile acid synthesis, pool size, and turnover. These results differ from those reported by Valdivieso et al. (30) and by Einarsson et al. (31), perhaps because the number of our subjects is small and the range in age was limited, possibly not great enough to allow the detection of differences.

We found major metabolic differences between subjects with radiolucent gallstones and controls. First, those with gallstones synthesized more cholesterol than controls and even when synthesis was inhibited by feeding cholesterol, it remained higher in gallstone subjects. Second, in response to increased dietary cholesterol, gallstone subjects, but not controls, secreted more cholesterol into bile. Third, on the regular or low-cholesterol diet, the rate of bile acid synthesis did not differ between groups, but in response to dietary cholesterol bile acid synthesis and pool decreased in women with gallstone. Bile acid pool size was smaller and FTR of both primary bile acids was faster in gallstone subjects on both diets.

Biliary lipids did not differ clearly between controls and gallstone subjects on either diet. The molar percent cholesterol in stimulated (gallbladder) bile was slightly higher in the gallstone group on the high cholesterol intake ( 7.9 vs. $5.6 \%, P$ $=0.08$, Table IV), but there were no other differences that approached statistical significance.

A number of investigators have called attention to the frequent similarity of biliary lipids in normal and gallstone patients, emphasizing the importance of factors other than bile lithogenicity in gallstone formation (32). Although the rate of biliary cholesterol secretion is usually greater in patients with

Table VI. Effects of Dietary Cholesterol on Biliary Cholesterol

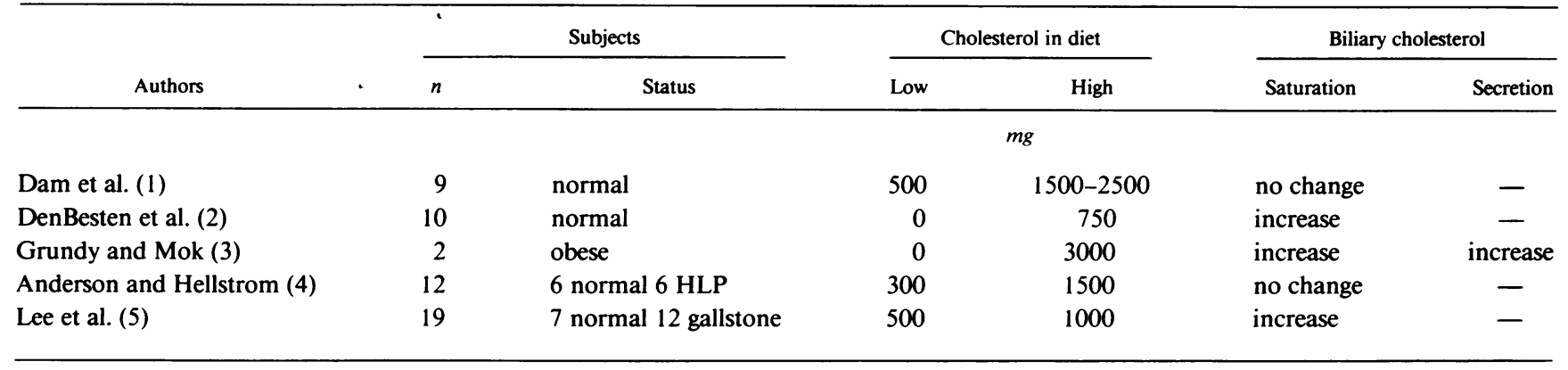

Abbreviation: HLP, hyperlipoproteinemia. 
gallstones, it was not in this small group of subjects. Biliary cholesterol secretion did increase, however, in gallstone subjects on the high cholesterol diet, but not in the controls. In fact, there were no effects of the high cholesterol diet on biliary lipids in the control subjects as noted in Table IV. Increases in dietary cholesterol of 500-750 mg/d caused an increase in CSI in two studies of normal subjects $(3,5)$, but in two other studies increases of $1,000-2,000 \mathrm{mg} / \mathrm{d}$ had no effect $(1,4)$. In our earlier study (6), an increase of $800 \mathrm{mg} / \mathrm{d}$ had no effect on CSI. The diet-induced increase in biliary cholesterol secretion in the gallstone subjects, but not in the controls, suggests that dietary cholesterol might be important in the pathogenesis of cholesterol gallstones and supports the hypothesis that hepatic metabolism of cholesterol in gallstone patients differs from those without stones.

Although the factors that regulate biliary cholesterol secretion are not known for certain, a number of studies have suggested that most biliary cholesterol is derived from preformed rather than newly synthesized cholesterol (33-39). Our finding of virtually identical specific activities of biliary and plasma cholesterol is consistent with that conclusion. Robins et al. (38) suggested that the precursor cholesterol for biliary secretion is transported directly from plasma through the plasma membrane of the liver cell to the bile canaliculus without entering the interior of the cell. On the other hand, Nervi et al. (39) found a reciprocal relationship between secretion of cholesterol in VLDL particles and in the bile, suggesting regulation by intracellular mechanisms. These, and other investigators, believe that the size of metabolic "pools" or compartments determines the fate of hepatic cholesterol.

Mean cholesterol synthesis was $44 \%$ greater in the gallstone subjects, with little overlap between groups $(P<0.001)$ (Fig. $2)$. As noted earlier $(6,18)$, the method used to measure cholesterol synthesis, the incorporation of labeled acetate into sterols by peripheral blood mononuclear cells, is a valid reflection of the relative rate of hepatic cholesterol synthesis under all circumstances studied, which include starvation, cholesterol feeding, cholestyramine and lovastatin administration, type II familial hypercholesterolemia, etc. The rate of cholesterol synthesis is known to increase with body mass index, as it did in the gallstone subjects in this study, but there was no difference in BMI or body weight between the groups. It can be concluded, therefore that the cholesterol synthesis rate is greater in individuals with radiolucent gallstone. Although cholesterol synthesis was diminished by the high cholesterol diet in both groups, it remained greater in the gallstone group, suggesting a basic difference in the regulation of cholesterol metabolism.

The activity of hepatic 3-hydroxy-3-methylglutaryl CoA reductase, the rate-limiting enzyme in cholesterol synthesis, has been studied in gallstone and control patients by six laboratories since 1975 (40-47) (Table VII). Although all used liver tissue obtained at surgery and essentially the same assay techniques, the reported results varied: four groups of investigators reported significantly greater activity in subjects with cholesterol gallstones than in those without stones $(40,41,43,44)$; one group found no change (42); the sixth group, in three studies in three groups of patients, found no change in two studies $(45,47)$ and a significant decrease in activity in the third study (46). The reason for the different results from this laboratory is not clear. Nervi et al. (48) measured cholesterogenesis from labeled acetate by liver slices obtained at surgery in normal weight gallstone and control subjects. They found higher cholesterol synthesis in both male and female gallstone subjects, but their groups were small, six in each group, and the intragroup differences were wide (sixfold) and not statistically significant. Thus, most of the published studies support the conclusion that cholesterol synthesis is higher in gallstone patients than in controls. Although increased cholesterogenesis is consistent with the observation that hepatic cholesterol, especially cholesterol ester, is increased in gallstone patients (40, 41 ), its role, if any, in the pathogenesis of cholesterol gallstones is not known.

3-Hydroxy-3-methylglutaryl CoA reductase activity in the liver and other tissues, including peripheral blood monocytes, is regulated by cellular uptake of cholesterol in chylomicron remnants and other lipoprotein fractions, especially LDL, and by the concentration of certain hydroxylated sterols, by-products of cholesterol synthesis, which inhibit cholesterol synthesis (49). Neither LDL receptor activity nor hydroxy sterol production was measured in these subjects, but plasma LDL levels, a major determinant of $\mathrm{LDL}$ receptor activity, and remnant uptake, measured by retinyl palmitate clearance, were the same in gallstone and control subjects. Although the amount of cholesterol absorbed in the gallstone subjects was slightly less than in the controls on both diets (Fig. 1), the difference appears too small to account for such a large difference in cholesterol synthesis on the basis of diminished feedback inhibition. This mechanism cannot be excluded, how-

Table VII. Hepatic 3-Hydroxy-3-methylglutaryl Coenzyme A Reductase Activity in Patients with Cholesterol Gallstones

\begin{tabular}{|c|c|c|c|c|c|c|c|c|}
\hline \multirow[b]{2}{*}{ Author } & \multirow[b]{2}{*}{ Year } & \multicolumn{2}{|c|}{ Controls } & \multicolumn{2}{|c|}{ Gallstones } & \multicolumn{3}{|c|}{ Reductase* } \\
\hline & & $n$ & \%IBW & $n$ & \%IBW & Control & GS & $P$ \\
\hline Salen et al. (40) & 1975 & 9 & 108 & 12 & 107 & 61 & 77 & 0.01 \\
\hline Coyne et al. (41) & 1976 & 4 & $?$ & 6 & $?$ & 113 & 153 & 0.01 \\
\hline Carulli et al. (42) & 1980 & 17 & 89 & 25 & 102 & 58 & 63 & NS \\
\hline Maton et al. (43) & 1980 & 10 & 92 & 6 & 124 & 14 & 32 & 0.001 \\
\hline Key et al. (44) & 1980 & 6 & 105 & 12 & 121 & 113 & 220 & 0.01 \\
\hline Ahlberg et al. (45) & 1981 & 10 & $104^{\ddagger}$ & 23 & $105^{\ddagger}$ & 25 & 21 & NS \\
\hline Angelin et al. (46) & 1982 & 10 & $155^{\ddagger}$ & 18 & $100^{\ddagger}$ & 61 & 38 & 0.05 \\
\hline Reihner et al. (47) & 1991 & 13 & $95^{\ddagger}$ & 22 & $97^{\ddagger}$ & 109 & 104 & NS \\
\hline
\end{tabular}

Abbreviations: GS, gallstones; IBW, ideal body weight. ${ }^{*}$ Activity $=\mathrm{pmol} / \mathrm{mg}$ microsomal protein $/ \mathrm{min} .{ }^{\ddagger}$ Relative body weight $((\mathrm{kg} / \mathrm{ht}[\mathrm{cm}]$ $-100) \times 100)$. 
ever, and should be explored in future studies. It is possible that the synthesis of inhibitory hydroxylated sterols, such as 25 - or 26-OH-cholesterol, a normal by-product of cholesterol synthesis, is less in the gallstone subjects. Little is known of the regulation of the synthesis of these sterols, but recent studies suggest that they serve as substrates for cholesterol $7-\alpha$ hydroxylase, the rate limiting enzyme in bile acid synthesis $(49,50)$. In summary the mechanism underlying a more rapid rate of cholesterol synthesis in gallstone patients is not known.

Bile acid kinetics. On a regular or low-cholesterol diet the gallstone subjects had more rapid FTR $(P<0.05)$ and smaller $(P=$ NS) bile acid pools (Table V), as has been described repeatedly $(2,6)$ since Vlahcevic and co-workers first reported it in 1971 (51). The cause of these kinetic differences has been debated, but not satisfactorily explained. The finding of similar rates of bile acid synthesis is consistent with earlier studies (51). Cholesterol 7- $\alpha$ hydroxylase activity in microsomes isolated from liver biopsy tissue was reported to be lower in gallstone patients than in controls in some studies $(40,41,43)$ but not in all (47). The effect of dietary cholesterol on bile acid kinetics in such patients has not previously been studied. In normal subjects, and in rats, a modest increase in dietary cholesterol has little or no effect on bile acid synthesis, as in our controls, whereas a very large increase in cholesterol intake may increase synthesis of bile acids in both species $(26,52-$ 54 ). The decrease in synthesis and pool in the gallstones subjects ingesting high cholesterol was not anticipated, but there is a rational biologic explanation, proposed below.

It is generally believed that the fate of hepatic free cholesterol is largely determined by the size of various intracellular metabolic pools or functional compartments of free cholesterol. As noted above, cholesterol for biliary secretion originates predominantly from preformed cholesterol. On the other hand, a number of lines of evidence suggest that $30-40 \%$ of the substrate for bile acid synthesis, regulated by the rate-limiting enzyme, cholesterol $7 \alpha$-hydroxylase, is derived from the intrahepatic synthesis of cholesterol.

The FTR of the bile acids did not change on the high cholesterol diet and the synthesis rate decreased; thus the bile acid pool must necessarily diminish, according to classical isotope dilution kinetics which states that synthesis rate equals pool multiplied by FTR. (Therefore, pool = synthesis $\div$ FTR.) When synthesis decreases without a change in turnover rate, pool decreases, accounting for the decreased bile acid pool in gallstone patients, especially those fed an increased amount of dietary cholesterol.

In summary, the metabolic responses to dietary cholesterol in patients with cholesterol gallstones observed in this study, the increase in biliary cholesterol secretion and decrease in bile acid synthesis, are internally consistent and provide a rational, physiologically sound basis for the secretion of supersaturated bile that is a necessary requisite for gallstone formation. Furthermore, they support the epidemiologic studies linking dietary cholesterol to gallstones and strongly support the contention that there are major metabolic differences in cholesterol metabolism in patients with gallstones.

\section{Acknowledgments}

The author thanks Carol McKinley, R.N., for able assistance with the clinical studies, Radene Showalter for expert technical assistance, and Mary Lu Jones, R.D., for assistance with dietary control.
This work was supported by National Institutes of Health grant R01 DK31765. Additional support for these studies was provided by the Hepatobiliary Research Center of the University of Colorado School of Medicine (National Institutes of Health grant 5P30 DK34914) and the General Clinical Research Center Program of the Division of Research Resources, National Institutes of Health (grant 5MR01 RR00051).

\section{References}

1. Dam, H., I. Prange, M. K. Jensen, H. E. Kallehauge, and H. J. Fenger. 1971. Studies on human bile. IV influence of ingestion of cholesterol in the form of eggs on the composition of bile in healthy subjects. Z. Ernaehrungswiss. 10:178-187.

2. DenBesten, L., W. E. Connor, and S. Bell. 1973. The effect of dietary cholesterol on the composition of human bile. Surgery. 73:266-273.

3. Grundy, S. M., and H. Y. I. Mok. 1979. Effect of diets and drugs on biliary cholesterol secretion in man. In Gallstones. M. M. Fisher, C. A. Goresky, E. A. Shaffer, and F. M. Strasbert, editors. Plenum Publishing Corp, New York. 238298.

4. Andersen, E., and K. Hellstrom. 1979. The effect of cholesterol feeding on bile acid kinetics and biliary lipids in normolipidemic and hypertriglyceridemic subjects. J. Lipid Res. 20:1020-1027.

5. Lee, D. W. T., C. J. Gilmore, G. Bonorris, H. Cohen, J. W. Marks, M. Cho-Sue, M. S. Meiselman, and L. J. Schoenfield. 1985. Effect of dietary cholesterol on biliary lipids in patients with gallstones and normal subjects. Am. J. Clin. Nutr. 42:414-420.

6. Everson, G. T., C. McKinley, and F. Kern, Jr. 1991. Mechanisms of gallstone formation in women: effects of exogenous estrogen (Premarin ${ }^{\star}$ ) and dietary cholesterol on hepatic lipid metabolism. J. Clin. Invest. 87:237-246.

7. Quintao, E., S. M. Grundy, and E. H. Ahrens, Jr. 1971. Effects of dietary cholesterol on the regulation of total body cholesterol in man. J. Lipid Res. 12:233-247.

8. Mistry, P., N. E. Miller, M. Laker, W. R. Hazzard, and B. Lewis. 1981. Individual variation in the effects of dietary cholesterol on plasma lipoproteins and cellular cholesterol homeostasis in man: studies of low density lipoprotein receptor activity and 3-hydroxy-3-methylglutaryl coenzyme A reductase activity in blood mononuclear cells. J. Clin. Invest. 67:493-502.

9. Grundy, S. M. 1983. Absorption and metabolism of dietary cholesterol. Annu. Rev. Nutr. 3:71-96.

10. Ahrens, E. H., Jr. 1984. After 40 years of cholesterol-watching. J. Lipid Res. 25:1442-1449.

11. Hegsted, D. M. 1986. Serum-cholesterol response to dietary cholesterol: a re-evaluation. Am. J. Clin. Nutr. 44:299-305.

12. McNamara, D. J., R. Kolb, T. S. Parker, H. Batwin, P. Samuel, C. D. Brown, and E. H. Ahrens, Jr. 1987. Heterogeneity of cholesterol homeostasis in man: response to changes in dietary fat quality and cholesterol quantity. J. Clin. Invest. 79:1729-1739.

13. Beynen, A. C., and M. B. Katan. 1985. Reproducibility of the variations between humans in the response of serum cholesterol to cessation of egg consumption. Atherosclerosis. 57:19-31.

14. Katan, M. B., A. C. Beynen, J. H. M. DeVries, and A. Nobels. 1986 Existence of consistent hypo- and hyperresponders to dietary cholesterol in man. Am. J. Epidemiol. 123:221-234.

15. Grundy, S. M., and M. A. Denke. 1990. Dietary influences on serum lipids and lipoproteins. J. Lipid Res. 31:1149-1172.

16. U. S. Department of Health and Human Services, Public Health Service. 1990. Healthy People 2000: National Health Promotion and Disease Prevention Objectives. Superintendent of Documents, Washington, DC.

17. White, E. C., D. J. McNamara, and E. H. Ahrens, Jr. 1981. Validation of a dietary record system for the estimation of the daily cholesterol intake in individual outpatients. Am. J. Clin. Nutr. 34:199-203.

18. Kern, F., Jr., and G. T. Everson. 1987. Contraceptive steroids increase cholesterol in bile: mechanisms of action. J. Lipid Res. 28:828-839.

19. Samuel, P., J. R. Crouse, and E. H. Ahrens, Jr. 1978. Evaluation of an isotope ratio method for measurement of cholesterol absorption in man. J. Lipid Res. 19:82-93.

20. McNamara, D. J., N. O. Davidson, and S. Fernandez. 1980. In vitro cholesterol synthesis in freshly isolated mononuclear cells of human blood: effect of in vivo administration of clofibrate and/or cholestyramine. J. Lipid Res. 21:65-71.

21. Berr, F., and F. Kern, Jr. 1984. Plasma clearance of chylomicrons labeled with retinyl palmitate in healthy human subjects. J. Lipid Res. 25:805-812.

22. Hazzard, W. R., and E. L. Bierman. 1976. Delayed clearance of chylomicron remnants following vitamin A-containing oral fat loads in broad- $\beta$ disease (type III hyperlipoproteinemia). Metab. Clin. Exp. 32:514-517. 
23. Grundy, S. M., and A. L. Metzger. 1972. A physiological method for estimation of hepatic secretion of biliary lipids in man. Gastroenterology. 62:1200-1217.

24. Everson, G. T., M. J. Lawson, C. McKinley, R. Showalter, and F. Kern, Jr. 1983. Gallbladder and small intestinal infusion of standard stimuli. J. Clin. Invest. 71:596-603.

25. Everson, G. T. 1987. Steady-state kinetics of serum bile acids in healthy human subjects: single and dual isotope techniques using stable isotopes and mass spectrometry. J. Lipid Res. 28:238-252.

26. Bennion, L. J., and S. M. Grundy. 1978. Risk factors for the development of cholelithiasis in man. N. Engl. J. Med. 299:1221-1227.

27. Miettinen, T. A. 1988. Regulation of serum cholesterol by cholesterol absorption. Agents Actions Suppl. 26:53-65.

28. Nestel, P. J., and A. Poyser. 1976. Changes in cholesterol synthesis and excretion when cholesterol intake is increased. Metab. Clin. Exp. 25:151-159.

29. Kern, F., Jr. 1991. Normal plasma cholesterol in an 88-year-old man who eats 25 eggs per day: mechanisms of adaptation. N. Engl. J. Med. 324:896-899.

30. Valdivieso, V., R. Palma, R. Wünkhaus, C. Antezana, C. Severín, and A. Contreras. 1978. Effect of aging on biliary lipid composition and bile acid metabolism in normal Chilean women. Gastroenterology. 74:871-874.

31. Einarsson, K., K. Nilsell, B. Leijd, and B. Angelin. 1985. Influence of age on secretion of cholesterol and synthesis of bile acids by the liver. N. Engl. J. Med. 313:277-282.

32. Holzbach, R. T., M. Marsh, M. Olszewski, and K. Holan. 1973. Cholesterol solubility in bile. Evidence that supersaturated bile is frequent in healthy man. J. Clin. Invest. 52:1467-1479.

33. Gregory, D. H., L. Swell, C. C. Schwartz, L. G. Halloran, and Z. R. Vlahcevic. 1978. Preferential utilization of free cholesterol secretion in man. Science (Wash. DC). 200:62-64.

34. Schwartz, C. C., M. Berman, Z. R. Vlahcevic, L. G. Halloran, D. H. Gregory, and L. Swell. 1978. Multicompartmental analysis of cholesterol metabolism in man. Characterization of the hepatic bile acid and biliary cholesterol precursor sites. J. Clin. Invest. 61:408-423.

35. Turley, S. D., and J. M. Dietschy. 1979. Regulation of biliary cholesterol output in the rat: dissociation from the rate of hepatic cholesterol synthesis, the size of the hepatic cholesteryl ester pool, and the hepatic uptake of chylomicron cholesterol. J. Lipid Res. 20:923-934.

36. Robins, S. J., and H. Brunengraber. 1982. Origin of biliary cholesterol and lecithin in the rat: contribution of new synthesis and preformed hepatic stores. $J$. Lipid Res. 23:604-608.

37. Robins, S. J., J. M. Fasulo, R. LeDuc, and G. M. Patton. 1989. The transport of lipoprotein cholesterol into bile: a reassessment of kinetic studies in the experimental animal. Biochim. Biophys. Acta. 1004:327-331.

38. Robins, S. J., J. M. Fasulo, M. A. Collins, and G. M. Patton. 1985. Evidence for separate pathways of transport of newly synthesized and preformed cholesterol into bile. J. Biol. Chem. 260:6511-6513.

39. Nervi, F., I. Marinovic, A. Rigotti, and N. Ulloa. 1988. Regulation of biliary cholesterol secretion: functional relationship between the canalicular and sinusoidal cholesterol secretory pathways in the rat. J. Clin. Invest. 82:18181825
40. Salen, G., G. Nicolau, S. Shefer, and E. H. Mosbach. 1975. Hepatic cholesterol metabolism in patients with gallstones. Gastroenterology. 69:676-684.

41. Coyne, M. J., G. G. Bonorris, L. I. Goldstein, and L. J. Schoenfield. 1976 Effect of chenodeoxycholic acid and phenobarbital on the rate-limiting enzymes of hepatic cholesterol and bile acid synthesis in patients with gallstones. J. Lab. Clin. Med. 87:281-291.

42. Carulli, N., M. PonzDeLeon, F. Zironi, A. Pinetti, A. Smerieri, R. Loria and $P$. Loria. 1980. Hepatic cholesterol and bile acid metabolism in subjects with gallstones: comparative effects of short term feeding chenodeoxycholic and ursodeoxycholic acid. J. Lipid. Res. 21:35-43.

43. Maton, P. N., H. J. Ellis, M. J. P. Higgins, and R. H. Dowling 1980. Hepatic HMGCoA reductase in human cholelithiasis: effects of chenodeoxycholic and ursodeoxycholic acids. Eur. J. Clin. Invest. 10:325-332.

44. Key, P. H., G. G. Bonorris, J. W. Marks, A. Chung and L. J. Schoenfield 1980. Biliary lipid synthesis and secretion in gallstone patients before and during treatment with chenodeoxycholic acid. J. Lab. Clin. Med. 95:816.

45. Ahlberg, J., B. Angelin, and K. Einarsson. 1981. Hepatic 3-hydroxy-3methylglutaryl coenzyme $A$ reductase activity and biliary lipid composition in man: relation to cholesterol gallstone disease and effects of cholic acid and chenodeoxycholic acid treatment. J. Lipid Res. 22:410-422.

46. Angelin, B., L. Backman, K. Einarsson, L. Eriksson, and S. Ewerth. 1982. Hepatic cholesterol metabolism in obesity: activity of microsomal 3-hydroxy-3 methylglutaryl coenzyme A reductase. J. Lipid Res. 23:770-773.

47. Reihnér, E., B. Angelin, I. Björkhem, and K. Einarsson. 1991. Hepatic cholesterol metabolism in cholesterol gallstone disease. J. Lipid Res. 32:469-475.

48. Nervi, F. O., C. F. Covarrubias, V. D. Valdivieso, B. O. Ronco, A. Solari and J. Tocornal. 1981. Hepatic cholesterogenesis in Chileans with cholesterol gallstone disease: evidence for sex differences in the regulation of hepatic cholesterol metabolism. Gastroenterology. 80:539-545.

49. Rudney, H., and R. C. Sexton. 1986. Regulation of cholesterol biosynthesis. Annu. Rev. Nutr. 6:245-272.

50. Dueland, S., J. D. Trawick, M. S. Nenseter, A. A. MacPhee, and R. A Davis. 1992. Expression of 7Alpha-hydroxylase in non-hepatic cells results in liver phenotypic resistance of the low density lipoprotein receptor to cholesterol repression. J. Biol. Chem. 267:22695-22698.

51. Vlahcevic, Z. R., J. R. Miller, J. T. Farrar, and L. Swell. 1971. Kinetics and pool size of primary bile acids in man. Gastroenterology. 61:85-90.

52. Reihner, E., I. Bjoerkhem, B. Angelin, S. Ewerth, and K. Einarsson. 1989. Bile acid synthesis in humans: regulation of hepatic microsomal cholesterol 7Alpha-hydroxylase activity. Gastroenterology. 97:1498-1505.

53. Grogan, W. M., M. L. Bailey, D. M. Heuman, and Z. R. Vlahcevic. 1991 Effects of perturbations in hepatic free and esterified cholesterol pools on bile acid synthesis, cholesterol $7 \alpha$-hydroxylase, HMG-CoA reductase, acyl-CoA:cholesterol acyltransferase and cytosolic cholesteryl ester hydrolase. Lipids. 26:907914.

54. Lin, D. S., and W. E. Connor. 1980. The long term effects of dietary cholesterol upon the plasma lipids, lipoproteins, cholesterol absorption, and the sterol balance in man: the demonstration of feedback inhibition of cholesterol biosynthesis and increased bile acid excretion. J. Lipid Res. 21:1042-1052. 